\section{MS32-P13 Agomelatine Phosphate: Salt or Co-Crystal?}

Eliška Skořepová ${ }^{1}$, Michal Hušák ${ }^{1}$, Luděk Ridvan ${ }^{2}$, Tereza Hanyková $^{1}$

1. Department of Solid State Chemistry, University of Chemistry and Technology Prague, Technická 5, 166 28, Praha 6, Czech Republic

2. Solid State Development, Zentiva k.s., Czech Republic

email: eliska.skorepova@vscht.cz

The search for new solid forms of an active pharmaceutical ingredient (API) is an important step in a drug development. Often, an API has a low water solubility, which then leads to a low oral bioavailability. The problem can be solved by crystallizing the API together with another chemical, resulting either in salt or co-crystal formation. Salts and co-crystal are multicomponent solids but in different ionization states. In salts, there is a proton transfer between the molecular components, making it contain cations and anions. On the other hand, co-crystals are made up from neutral molecules held together by non-bonded interactions. One such API is agomelatine (AG), a melatonergic antidepressant. However, agomelatine is an amidic compound and, since amides are generally considered neutral and, for agomelatine, only co-crystals have been published, it was quite a surprise, when agomelatine, in the combination with phosphoric acid, produced a salt. Moreover, the $\Delta \mathrm{pK}$ calculation clearly indicated a co-crystal preference. ${ }^{\text {a }}$ However, we were able to obtain synchrotron single-crystal diffraction data and solve the structure. The position of the acidic hydrogen was located in the difference Fourier map. Specifically, the amide oxygen was protonated. The proton transfer and the salt formation were also confirmed by solid state NMR and through CASTEP energy minimization. For pharmaceuticals, the determination whether the material is a salt or a co-crystal is interesting not only academically, but also from the regulatory point of view. Therefore, our findings may play a crucial role in the future development of the multicomponent solid phases of agomelatine.

This work was supported by the Grant Agency of Czech Republic, Grant no. 106/14/03636S and received financial support from specific university research (MSMT No 20/2016). We acknowledge the ESRF for provision of synchrotron radiation facilities and we would like to thank J. Wright for assistance in using beamline ID11.

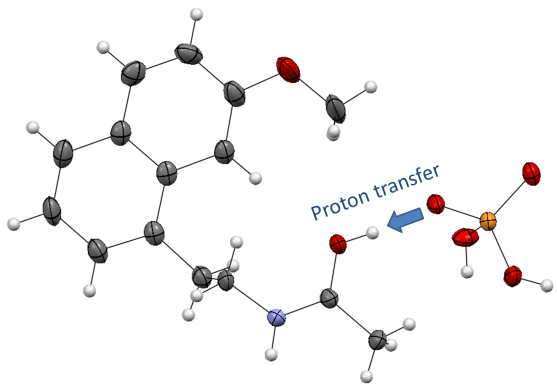

Keywords: pharmaceuticals, salt, co-crystal, $\Delta \mathrm{pKa}$, crystal structure, proton transfer

Figure 1. The asymmetric unit of agomelatine phosphate 